Punkte-Sammeln jetzt noch komfortabler!

\title{
CME.springer.de wird zur e.Akademie
}

Die e.Akademie von Springer Medizin ist die Weiterentwicklung von CME.springer.de und bietet Ihnen ein noch umfassenderes und moderneres Fortbildungsangebot: Mehr als 1500 Fortbildungsmodule, neue e.Learningformate und multimediale Elemente machen Ihre Fortbildung und das Sammeln von CME-Punkten noch flexibler und komfortabler.

e.CME: Als Zeitschriftenabonnent stehen Ihnen in der e.Akademie von Springer Medizin nach wie vor alle zertifizierten Fortbildungsbeiträge Ihrer Zeitschrift als e.CME (PDF des Artikels plus OnlineFragebogen) zur Verfügung. Darüber hinaus haben Sie künftig die Möglichkeit, Beiträge Ihrer Zeitschrift, deren Zertifizierungszeitraum abgelaufen ist, weiterhin für Ihre Fortbildung und persönlichen Wissenscheck zu nutzen.

Auch als Leser einer Zeitschrift können Sie die Fortbildung des jeweils aktuellen Heftes kostenfrei nutzen - allerdings nur für einen begrenzten Zeitraum. Dazu benötigen Sie die jeweils gültige Fortbildungsidentifikationsnummer (FIN), wie im Kasten rechts erläutert.

\section{e.Med-Abonnenten \\ profitieren vom vollen Umfang}

Nutzer von e.Med, dem Springer Medizin Online-Paket, profitieren vom vollen Leistungsumfang der e.Akademie: Mehr als 1500 e.CMEs aus den wichtigsten medizinischen Fachgebieten werden ergänzt durch die neuen e.Learningformate e.Tutorial und e.Tutorial plus. Das e.Tutorial ist speziell für die Online-Fortbildung konzipiert und didaktisch optimiert. Klar gegliederte Lernabschnitte, besondere Hervorhebung von Merksätzen, zoomfähige Abbildungen und Tabellen sowie verlinkte Literatur erleichtern das Lernen und den Erwerb von CME-Punkten.

\section{e.Tutorial.plus:}

\section{Fortbildung vom Feinsten}

Das e.Tutorial.plus bietet multimedialen Zusatznutzen in Form von Audio- und Videobeiträgen, 3D-Animationen, Experteninterviews und weiterführende Informationen. CME-Fragen und Mul-
tiple-Choice-Fragen innerhalb der einzelnen Lernabschnitte ermöglichen die Lernerfolgskontrolle.

I) Der direkte Weg zur e.Akademie unter:

springermedizin.de/eAkademie

॥) Weitere Informationen zum e.MedKomplettpaket und Gratis-Testangebot unter: springermedizin.de/ eMed

\section{CME \\ Mit FIN punkten Leser kostenfrei!}

\section{Registrierung und Teilnahmemöglichkeiten}

Falls Sie noch keinen Springer-Medizin-Zugang haben, bitten wir Sie, sich einmalig auf www.springermedizin.de zu registrieren.

- e.CME: Kostenfreie Teilnahme bis zum 16. Mai 2012

Gehen Sie auf springermedizin.de/eAkademie und geben Sie die achtstellige Fortbildungsidentifikationsnummer (FIN) des Beitrags in die Suchmaske ein. Sie gelangen damit direkt zum gewünschten e.CME-Beitrag. Die FIN für diesen Beitrag lautet: AD1202qS

- e.Tutorial: Teilnahme im Rahmen des e.Med-Abonnements Weitere Infos auf springermedizin.de/eMed

Bitte beachten Sie:

- Teilnahme nur online unter springermedizin.de/eAkademie

- Die Frage-Antwort-Kombinationen werden online individuell zusammengestellt

- Es ist immer nur eine Antwort möglich

- Nach erfolgreicher Beantwortung von mindestens 7 der 10 Fragen erlangen Sie 3 CME-Punkte. Diese werden direkt an Ihre Landesärztekammer übertragen, bei Bedarf können Sie sich auch eine Bescheinigung ausdrucken

\section{Kontakt und weitere Informationen:}

Springer-Verlag GmbH

Tel.: 08007780777
Springer Medizin Kundenservice

E-Mail: kundenservice@springermedizin.de 